\title{
Accuracy of Ratio for Center of The Ankle Method as a Landmark for Proximal Tibial Resection in Computer Assisted Total Knee Arthroplasty Compared with Extramedullary Method
}

\author{
Anuchit Vejjaijiva*, Pornpavit Sriphirom, Chalit Saepoo, \\ Weeravut Ounjitti and Soros Suwansawaiphol \\ Rajavithi Hospital, Rangsit University, Bangkok, Thailand \\ joyanuchitegmail.com, pornpaviteyahoo.com, \\ bell_ass@hotmail.com, oun_ang_krub@outlook.com, \\ sorosgap@gmail.com
}

\begin{abstract}
Proximal tibial resection is an important surgical step in total knee arthroplasty (TKA). Normally, an anterior surface of tibia used as anatomical landmark (extramedullary method), this method based on visual judgment. (D.C. Marchant, 2005) calculated ratio for center of the ankle, ratio of lateral distance to total inter-malleolar distance was observed at 0.57 in normal ankle sample. However, there is no study documenting about accuracy of ratio for center of the ankle as a landmark for proximal tibial resection in computer assisted TKA (CAS TKA).

This was prospective study on 65 patients with osteoarthritis knees scheduled to receive CAS TKA from January to December 2018. Proximal tibial resection was simulated by extramedullary method and ratio for center of the ankle respectively. Tibial resection alignment were recorded by CAS.

Tibial resection alignment within 3 degrees was 84.6 and $92.3 \%$ for extramedullary method and ratio for center of the ankle method. The average total tibial resection alignment was 0.84 and 0.66 degrees of valgus $(p=0.497)$, the average tibial resection alignment within 3 degrees from mechanical axis was 0.33 and 0.55 degrees of valgus ( $p$ $=0.513$ ) and the average tibial resection alignment more than 3 degrees from mechanical axis was 3.60 and 1.5 degrees of valgus $(p=0.049)$ for extramedullary method and ratio for center of the ankle method respectively.

Ratio for center of the ankle demonstrated result in numeric value and anatomical landmark of lateral and medial malleoli was simple to identified. Our results shown the
\end{abstract}


tibial resection alignment with ratio for center of the ankle method had higher accuracy and lower average degrees of outlier than extramedullary method.

\section{Introduction}

Proximal tibial resection is an important surgical step in total knee arthroplasty (TKA). Normally, an anterior surface of tibia used as anatomical landmark (extramedullary method), this method based on visual judgment. DA Dennis et al. ${ }^{1}$ showed $88 \%$ of extramedullary method were aligned within $2^{\circ}$ of $90^{\circ}$ goal versus only $72 \%$ of intramedullary method. Bruno et al. ${ }^{2}$ showed the mean alignment of the tibial component was $90.3^{\circ}\left(84-97^{\circ}\right)$ with intramedullary method and $88.5^{\circ}\left(83-94^{\circ}\right)$ in extramedullary method. Tsukeoka et al. ${ }^{3}$ they concluded rotational mismatch between the proximal and distal part of the tibia should be avoided and distance between the alignment rod and the bone should be as short as possible to achieve proper tibial alignment. Extramedullary method had a good accurate but some literatures reported many factors might be affect the accuracy of extramedullary method such as preoperative leg's deformity, length and width of the leg, tibial bowing and parallax error.

Tanutum Pikulkaew er al. ${ }^{4}$ reported center of the ankle in THAI subjects were located lateral to the mechanical axis with an average of $2.1 \pm 1.1 \mathrm{~mm}$. D.C. Marchant et al..$^{5}$ calculated ratio for center of the ankle, ratio of lateral distance to total inter-malleolar distance was observed at 0.57 in normal ankle sample. Therefore, a thorough understanding of corrected landmark for proximal tibial cutting is critical for successful TKA. The aim of this study was to investigate the accuracy of ratio for center of the ankle as a landmark for proximal tibial resection in computer assisted TKA (CAS TKA).

\section{Materials and Methods}

We prospective reviewed 65 patients with osteoarthritis knees undergoing primary CAS TKA (Orthopilot 4.4, B. Braun, Aesculap, Tuttlingen, Germany) from January to December 2018. Proximal tibial resection was simulated by extramedullary method and ratio for center of the ankle method respectively. Tibial resection alignment were recorded by CAS and compared between both methods.

First, we simulated proximal tibial resection with extramedullary method by placed the cutting guide instrument to parallel with anterior $1 / 3$ of tibial crest. Second, we measured intermalleolar distance from the most prominent point at lateral and medial malleolus then marked the point at $57 \%$ from lateral malleolus as a landmark for placed the cutting guide instrument onto this area (ratio for center of the ankle method). Information regarding tibial cutting alignment were collected from the navigation data for analysis.

\section{Results}

Tibial resection alignment within 3 degrees was 84.6 and $92.3 \%$ for extramedullary and ratio for center of the ankle. The average total tibial resection alignment was 0.84 and 0.66 degrees of valgus ( $p$ $=0.497$ ), the average tibial resection alignment within 3 degrees from mechanical axis was 0.33 and 0.55 degrees of valgus $(\mathrm{p}=0.513)$ and the average tibial resection alignment more than 3 degrees from mechanical axis was 3.60 and 1.5 degrees of valgus $(p=0.049)$ for extramedullary method and ratio for center of the ankle method respectively. 


\section{Discussions}

Although extramedullary method had a good accuracy but some literatures reported many factors might be affect the accuracy of extramedullary method. Ratio for center of the ankle demonstrated result in numeric value and anatomical landmark of lateral and medial malleoli was simple to identified. Our results shown the tibial resection alignment with ratio for center of the ankle method had higher accuracy and lower average degrees of outlier than extramedullary method. This landmark guided the surgeons know the true center of the ankle easier than visual judgement with extramedullary method. We concluded, ratio for center of the ankle is one of accurate, reproducible and reliable anatomy landmark for proximal tibial resection in TKA.

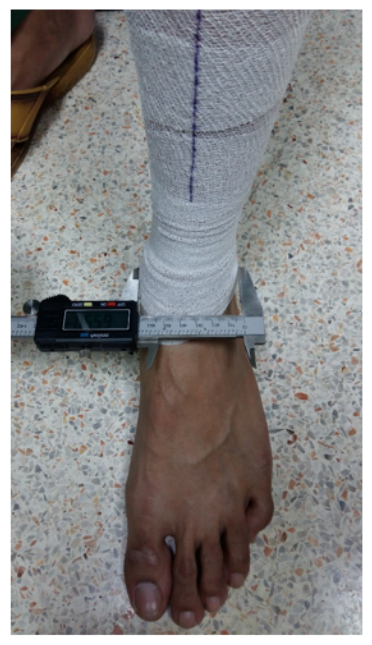

Intermalleolar distance

$(100 \%)$

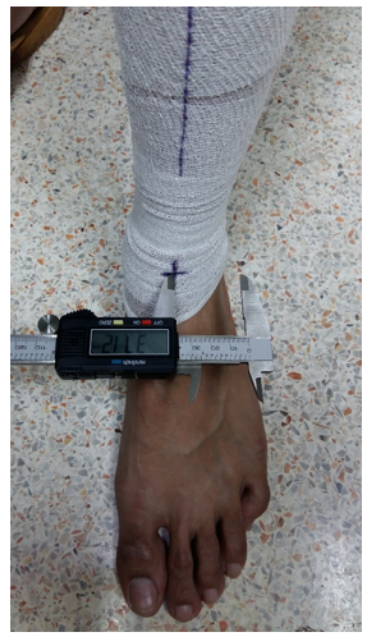

Marked point at $57 \%$ from lateral malleolus

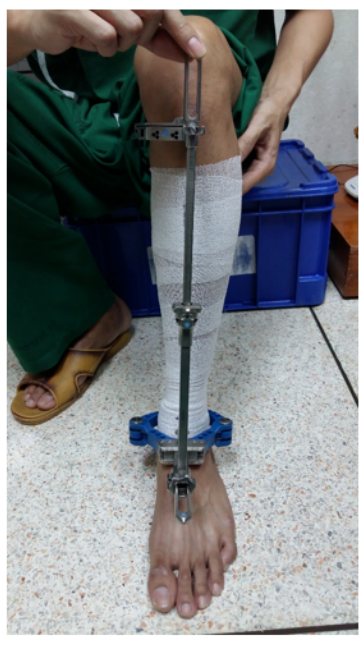

Placed the instrument on marking area

Figure 1: Ratio for center of the ankle method 


\begin{tabular}{lc}
\hline & $\begin{array}{c}\text { Percent of tibial resection within } 3^{\circ} \\
\text { from mechanical axis }\end{array}$ \\
\hline Extramedullary Method & $84.6 \%$ \\
Ratio for center of the ankle Method & $92.3 \%$ \\
\hline
\end{tabular}

Table 1: Results in tibial resection alignment

\begin{tabular}{|c|c|c|c|}
\hline & $\begin{array}{l}\text { Extramedullary } \\
\text { Method }\end{array}$ & $\begin{array}{l}\text { Ratio for center of } \\
\text { the ankle Method }\end{array}$ & P-value \\
\hline $\begin{array}{l}\text { Average total tibial } \\
\text { resection } \\
\text { alignment }\end{array}$ & $0.84^{\circ}$ valgus & $0.66^{\circ}$ valgus & 0.497 \\
\hline $\begin{array}{l}\text { Average tibial } \\
\text { resection } \\
\text { alignment within } \\
3^{\circ} \text { from } \\
\text { mechanical axis }\end{array}$ & $0.33^{\circ}$ valgus & $0.55^{\circ}$ valgus & 0.513 \\
\hline $\begin{array}{l}\text { Average tibial } \\
\text { resection } \\
\text { alignment }>3^{\circ} \\
\text { from mechanical } \\
\text { axis }\end{array}$ & $3.60^{\circ}$ valgus & $1.50^{\circ}$ valgus & 0.049 \\
\hline
\end{tabular}

Table 2: Sub-analysis in tibial resection alignment

\section{References}

1. Dennis DA, Channer M, Susman MH, Stringer EA. Intramedullary versus extramedullary tibial alignment systems in total knee arthroplasty. J Arthroplasty. 1993 Feb;8(1):43-7.

2. Bruno da Rocha Moreira Rezende, Thiago Fuchs, Rodrigo Nishimoto Nishi, Munif Ahmad Hatem, Luciana Mendes Ferreira da Silva, Rogerio Fuchs, Paulo Gilberto Cimbalista de Alencar. Alignment of the tibial component in total knee arthroplasty procedures using an intramedullary or extramedullary guide: double-blind randomized prospective study. Rev Bras Ortop. 2015 Mar-Apr; 50(2):168-173.

3. Tadashi Tsukeoka, Yoshikazu Tsuneizumi, Tae Hyun Lee. The effect of rotational fixation error of the tibial cutting guide and the distance between the guide and the bone on the tibial osteotomy in total knee arthroplasty. J Arthroplasty. 2013 Aug;28(7):1094-1098.

4. Tanutum Pikulkaew, Chaturong Pornrattanamaneewong, Krrrati Chareancholvanich. Anatomical landmark to locate the ankle center for determination of the mechanical axis of the lower extremity in Thai subjects. J Med Assoc Thai 2012; 95(suppl. 9):S1-S5

5. Marchant DC, Rimington DP, Crawford RW. An algorithm for locating the center of the ankle joint in knee navigation surgery. Computer Aided Surgery. January 2005;10(1):45-49. 